\title{
Etiologic Factors of The Periodontal Disease Pathogenesis
}

\author{
Ambarkova Vesna* \\ Department for Preventive and Pediatric Dentistry, University Ss Cyril \& Methodius, Macedonia \\ *Corresponding author: Vesna Ambarkova, Department of Paediatric and Preventive Dentistry, University St. Cyril and Methodius, Faculty of Dental \\ Medicine, Mother Theresa 17 University Dental Clinic Center Sv Pantelejmon, Skopje 1000, Macedonia
}

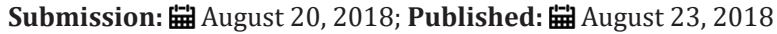

\section{Editorial}

Dental plaque bacteria along with its products such as lipopolysaccharides (LPS) cause the destruction of the periodontal tissue directly and indirectly by activating the host's defensive cells of the immune system, which in turn produce and release mediators that stimulate the effects of connective tissue expansion. Microbial plaque components have the capacity to induce initial inflammation and infiltration of inflammatory cells, including lymphocytes, macrophages, and polymorph nuclear leucocytes (PMNl). Microbial components, in particular LPS, activate macrophages to synthesize and secrete various proinflammatory molecules, including cytokines-IL-1 and tumor necrosis factor-alpha (TNF-alpha); prostaglandins, especially prostaglandin E2 (PGE2) and hydrolytic enzymes. The bacteria activate $\mathrm{T}$ lymphocytes for the production of IL-1 and lymphotoxin (LT), a molecule with similar properties of TNF-alpha. These cytokines manifest potent proinflammatory and catabolic activities and play a key role in the formation of periodontal pockets through collagenolytic enzymes such as matrix metalloproteinases (MMP). These latent collagenolytic enzymes are activated by reactive oxygen species in the inflammatory environment, leading to increased levels of interstitial collagenase in the inflamed gingiva, which deepens the gingival sulcus, creating a parodontal pocket [1]. A periodontal pocket with a depth of 4 to $12 \mathrm{~mm}$ is a port of 107 to 109 bacterial cells.

In addition to local action, the systemic effect of gram negative bacteria and LPS is a vascular response involving inflammatorycellular blood vessel infiltration, intravascular coagulation, vascular smooth muscle proliferation, and fat degeneration [2]. LPS increases the expression of endothelial cell adhesion and activate macrophages to synthesize and secrete various proinflammatory molecules and thromboxane, which leads to aggregation and platelet adhesion [3].

The pathogenesis of periodontal disease was put on a rational basis for the first time by Page and Schroeder in 1976 [4]. The destructive process is initiated by bacterial LPS, but further evolution depends on the host's immune system. In the presence of a microbial challenge, the host responds with immediate inflammatory and immune response in order to control the challenge [5]. This immune response to hominine, although initially directed against the bacteria, leads to perturbation of the local homeostasis and mediates the destruction of the periodontal tissue, leading to progression of the disease [6].

In the last few years, it has become clear that susceptibility to periodontal disease significantly varies among individuals having the same pathogenic microflora, and most of the evidence suggests that the host's immune response to the bacterial challenge is the major determinant of the disease development $[7,8]$. It is assumed that the destruction of the periodontal tissue is a consequence of excessive activation of the host's inflammatory response to bacterial infection leading to increased secretion of proinflammatory cytokines (IL-1beta, TNF alpha, IL-6, IL-17), MMP and PGE2 induced reduced production of IL-10 (interleukin-10), TGF-beta (transforming growth factor beta), which are tissue inhibitors of matrix metalloproteinases (MMP). From this it follows that the balance of produced cytokines regulates bone tissue homeostasis, progression, or regression of the disease $[9,10]$.

Over the years, various biologically plausible mechanisms have been established for a common inflammatory etiopathogenesis of Diabetes mellitus (DM) as metabolic disease characterized by an increased blood glucose level and periodontal disease which is mainly is mainly characterized by the destruction of tooth support tissues [11,12]. Numerous epidemiological studies have found a association between DM and periodontal disease and also and periodontal disease has even been proposed as a sixth complication of diabetes mellitus [12]. Today is clearly demonstrated that this relationship is bidirectional, the high prevalence of periodontal disease in DM indicates the need to evaluate glucose levels in periodontal patientsm while intervention studies have demonstrated that the treatment of periodontal disease improves the glycemic control of DM patients [12-14]. 
In the cross-sectional case-control study conducted by Buduneli et al. [15] with the aim to provide a comparative evaluation of clinical periodontal measurements, together with serum levels of certain bioactive peptides and inflammatory cytokines, in relation to obesity. The clinical periodontal measurements and the levels of serum leptin, adiponectin, interleukin-6 (IL-6), C-reactive protein and soluble intercellular adhesion molecule-1 of obese female individuals and their nonobese counterparts were compared [15]. The obesity group of examiners does not seem to have a prominent effect on clinical periodontal parameters but it does have many correlations with circulating inflammatory molecules like increased levels of leptin and IL- 6 might be one explanation for a possible relationship between obesity and periodontal disease [15].

The systematic review by Keller A et al. [16] suggests that overweight, obesity, weight gain, and increased waist circumference may be risk factors for development of periodontitis or worsening of periodontal measures [16]. Intervention and longitudinal studies with overweight or obesity as exposure and periodontitis as outcome were searched through the platforms PubMed/ Medline and Web of Knowledge. Two intervention studies on the influence of obesity on periodontal treatment effects found that the response to non-surgical periodontal treatment was better among lean than obese patients; the remaining three studies did not report treatment differences between obese and lean participants [16]. Interleukin (IL)-34 has recently been identified as an alternative ligand for colony stimulating factor-1 receptor and plays an important role in osteoclastogenesis. The aim of the study conducted by Guruprasad CN et all was to assess and compare IL34 levels in gingival crevicular fluid (GCF) and plasma in obese individuals in the presence or absence of periodontal disease and to determine whether they showed a correlation with disease severity [17]. The results of a clinico-biochemical study suggest that obese individuals with periodontitis have higher GCF and plasma IL-34 levels than non-obese individuals with healthy periodontium. This suggests IL-34 as a potential inflammatory marker of periodontal disease and obesity [17]. In the literature different life-course epidemiology theories which explain the possible association between periodontal health and nutritional status in adulthood could be found [18]. A prospective study are warranted to clarify, in greater detail, the effects of obesity on periodontal health.

Smoking cessation reduced the risk for periodontitis onset and progression and improved the outcomes of nonsurgical periodontal therapy. The findings of few studies have demonstrated that the risk for periodontitis becomes comparable to that of never-smokers and that nonsurgical periodontal treatment outcomes improve after smoking cessation. Dental professionals ought to consider smoking cessation interventions as a relevant component of the periodontal therapy [19]. Porphyromonas gingivalis (P gingivalis) and nicotine have been implicated as a major pathogen in the development and progression of periodontitis. The oxidative stress biomarkers were generated by human periodontal ligament fibroblasts (PDLF) upon treatment with periodontal pathogens and nicotine which could elucidate a potential local mechanism of periodontal disease etiology via superoxide mediation [20].
The body produces chromogranin A (ChgA) in response to stress as an adaptive reaction. Because of that ChgA is used as an index of autonomic nervous system activity, it is also involved in the immunomodulation system, and an increase in its production in patients with periodontal disease and cigarette smokers has been reported [21]. Sadaoka et al. [20] conclude from their study that human periodontal ligament-derived fibroblasts (HPdLF) produced ChgA, and nicotine increased ChgA production [21].

Accordingly, prospective studies are required to verify the impact of diabetes mellitus, obesity and use of tobacco on the development of chronic oral conditions. Such investigations could provide basis for the most appropriated time to start preventive strategies in order to reduce disease occurrence and saving public financial health resources and improving the quality of life of the individuals and population from the whole world.

\section{References}

1. Lee W, Aitken S, Sodek J, McCulloch CA (1995) Evidence of a direct relationship between neutrophil collagenase activity and periodontal tissue destruction in vivo: role of active enzyme in human periodontitis. J Periodontal Res 30(1): 23-33.

2. Mattila K (2003) Does periodontitis cause heart disease? Eur heart J 24: 2079-2081.

3. Herzberg MC, Meyer MW (1996) Effects of oral flora on platelets: possible consequences in cardiovascular disease. J Periodontol 67(10 suppl): 1138-1142.

4. Ekstein J, Shapira L, Van TE (1993) The pathogenesis of periodontal disease: a paradigm shift. Refuat Hapeh Vehashinayim 27(3): 35-39, 63.

5. Jotwani R, Cutler CW (2003) Multiple dendritic cell (DC) subpopulations in human gingiva and association of mature DCs with CD4+ T-cells in situ. J Dental Res 82(9): 736-741.

6. Garlet GP, Cardoso CR, Campanelli AP, Martins W, Silva JS (2006) Expression of suppressors of cytokine signaling in diseased periodontal tissues: a stop signal for disease progression? J Periodontal Res 41(6): 580-584.

7. Hajishengallis G (2014) Immunomicrobial pathogenesis of periodontitis: keystones, pathobionts, and host response. Trends Immunol 35(1): 3-11.

8. Hajishengallis G (2015) Periodontitis: from microbial immune subversion to systemic inflammation. Nat Rev Immunol 15(1): 30-44.

9. Bernardo ME, Fibbe WE (2013) Mesenchymal stromal cells: sensors and switchers of inflammation. Cell Stem Cell 13(4): 392-402.

10. Kayal RA (2013) The role of osteoimmunology in periodontal disease. Biomed Research International.

11. Ambarkova V (2018) Connection between the periodontal disease and diabetes mellitus. Interventions Obes Diabetes 1(5): 1-2.

12. Bascones A, González J, Sanz J (2014) Diabetes and periodontal disease. Review of the literature. Am J Dent 27(2): 63-67.

13. Stanko P, Izakovicova Holla L (2014) Bidirectional association between diabetes mellitus and inflammatory periodontal disease. A review. Biomed Pap Med Fac Univ Palacky Olomouc Czech Repub158(1): 35-83.

14. Wang TF, Jen IA, Chou C, Lei YP (2014) Effects of periodontal therapy on metabolic control in patients with type 2 diabetes mellitus and periodontal disease: a meta-analysis. Medicine (Baltimore) 93(28): e292.

15. Buduneli N, Bıyıkoğlu B, Ilgenli T, Buduneli E, Nalbantsoy A, et al. (2014) Is obesity a possible modifier of periodontal disease as a chronic inflammatory process? A case-control study. J Periodontal Res 49(4): 465-471. 
16. Keller A, Rohde JF, Raymond K, Heitmann BL (2015) Association between periodontal disease and overweight and obesity: a systematic review. J Periodontol 86(6): 766-776.

17. Guruprasad CN, Pradeep AR (2018) Interleukin-34 levels in gingival crevicular fluid and plasma in healthy and diseased periodontal tissue in presence or absence of obesity: a clinico-biochemical study. Bull Tokyo Dent Coll 59(2): 79-86.

18. Nascimento GG, Leite FR, Correa MB, Horta BL, Peres MA, et al. (2014) Relationship between periodontal disease and obesity: the role of lifecourse events. Braz Dent J 25(2): 87-89.
19. Leite FRM, Nascimento GG, Baake S, Pedersen LD, Scheutz F, et al. (2018) Impact of smoking cessation on periodontitis: A systematic review and meta-analysis of prospective longitudinal observational and interventional studies. Nicotine Tob Res.

20. Nguyen TT, Huynh NN, Seubbuk S, Nilmoje T, Wanasuntronwong A, et al. (2018) Oxidative stress induced by Porphyromonas gingivalis lysate and nicotine in human periodontal ligament fibroblasts. Odontology doi: 10.1007/s10266-018-0374-1.

21. Sadaoka S, Yagami K, Maki S (2013) Nicotine in cigarettes promotes chromogranin a production by human periodontal ligament fibroblasts. Arch Oral Biol 58(8): 1029-1033.
Creative Commons Attribution 4.0 International License

For possible submissions Click Here

\section{Submit Article}

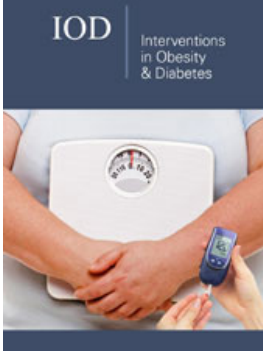

\section{Intervention in Obesity \& Diabetes}

\section{Benefits of Publishing with us}

- High-level peer review and editorial services

- Freely accessible online immediately upon publication

- Authors retain the copyright to their work

- Licensing it under a Creative Commons license

- Visibility through different online platforms 